\title{
Leben aus dem Geiste oder: Warum Bildungsein- richtungen als menschliche Entfaltung tragende ,Gemeinden' gedacht werden sollten
}

\begin{abstract}
Summary (Renate Girmes: Living Through the Spirit or: Why Educational Institutions Should Be Considered "Communities" for Human Development): The following text explores existing arguments for a new view on the tasks of the education system from a tradition of Christian thought. It takes up what can be considered the sense and promise of baptism in its argument for the endorsement of the Gospel of John in this tradition. A way of reading in this tradition is also developed, revealing what foundational information on the nature of intergenerational relationships can be found in the biblical text, and what connected challenges a professional education and learning praxis faces.
\end{abstract}

Keywords: Baptism, education, development, intergenerational relations, pedagogical professionalism

\begin{abstract}
Резюме (Ренате Гирмес: жизнь, вытекающая из образа мыслей, или: Почему образовательные учреждения должны быть задуманы как ,общины', способствующие человеческому развитию): Нижеследующий текст исследует, какие существуют аргументы для нового взгляда на задачи системы образования с точки зрения традиционного христианского мышления. Он исследует, что в этой традиции может рассматриваться как смысл и обетования крещения в их аргументированной опоре на Евангелие от Иоанна. Для этого разрабатывается толкование данной традиции, которое раскрывает, какие принципиальные сведения может дать библейский текст о формировании отношений поколений и каким связанным с этим вызовам противостоит профессиональная практика воспитания и образования.

Ключевые слова: Крещение, образование, раскрытие, отношение поколений, педагогический профессионализм

Zusammenfassung: Der nachfolgende Text lotet aus, welche Argumente es für eine neue Sicht auf die Aufgaben des Bildungssystems aus der Tradition christlichen Denkens gibt. Er greift auf, was in dieser Tradition als der Sinn und die Verheißung der Taufe in deren argumentativer Stützung auf das Johannesevangelium betrachtet werden kann. Dazu wird eine Lesart zu dieser Tradition entwickelt, die entfaltet, welche grundsätzliche Auskunft der biblische Text über die Gestaltung des Generationenverhältnisses zu geben vermag und welchen damit verbundenen Herausforderungen eine professionelle Erziehungs- und Bildungspraxis gegenübersteht.i
\end{abstract}

Schlüsselwörter: Taufe, Bildung, Entfaltung, Generationsverhältnis, pädagogische Professionalität

Es ist der 6. Sonntag nach dem Fest Trinitatis, zur Dreifaltigkeit Gottes, der sich mit der Taufe auseinandersetzt, die die Menschen im Kontext der Kirche zu einer Gemeinschaft im Geiste Gottes macht. Zur christlichen Reflexion über die Taufe gehört der nachfolgend abgedruckte Text aus dem Johannesevangelium:

„Es war aber unter den Pharisäern ein Mann mit Namen Nikodemus, ein Ratsherr der Juden. Dieser kam zu ihm bei Nacht und sagte zu ihm: „Rabbi, wir wissen, dass du als Lehrer von Gott gekommen bist. Denn niemand kann diese Zeichen wirken, wenn nicht Gott mit ihm ist.“ Jesus antwortete und sprach zu ihm: „Wahrlich, wahrlich, ich sage dir: Wer nicht von oben her geboren wird, kann das Reich Gottes nicht schauen." Nikodemus sagte zu ihm: „Wie kann ein Mensch geboren werden, wenn er ein Greis ist? 
Kann er etwa zum zweitenmal in den Schoß seiner Mutter eingehen und geboren werden?“ Jesus antwortete: „Wahrlich, wahrlich, ich sage dir: Wer nicht aus Wasser und Geist geboren wird, kann nicht in das Reich Gottes eingehen. Was aus dem Fleisch geboren ist, ist Fleisch; was aus dem Geist geboren ist, ist Geist. Wundere dich nicht darüber, daß ich zu dir sagte, ihr müßt von oben geboren werden. Der Wind weht, wo er will, und du hörst sein Sausen; aber du weißt nicht, woher er kommt noch wohin er geht. So verhält es sich mit jedem, der aus dem Geist geboren ist." Johannes 3, 1-8 (Übersetzung der Jerusalemer Bibel)

Die hier zum Taufkonzept beigezogene Passage widmet sich dem Mysterium des Geistes und betrachtet dessen Bedeutung im Leben der Menschen. Der von Gott gekommene Lehrer, nämlich Jesus, erläutert dieses Mysterium einem lehrenden Menschen, nämlich Nikodemus. Dieser erfährt, was es bedeutet, aus dem Geiste geboren zu sein/ zu werden und entsprechend zu handeln; er bekommt das gesagt, weil es zu wissen wichtig sei, wenn Menschen in das Reich Gottes eingehen können sollen, wozu ein Lehrer der Menschen ja seinen Beitrag zu leisten in der Lage sein sollte. Er sollte - so Jesus - deshalb lernen zu verstehen, dass aus dem Geist geboren zu werden und entsprechend zu leben/ handeln, bedeutet: "Der Wind weht, wo er will, und du hörst sein Sausen; aber du weißt nicht, woher er kommt noch wohin er geht. So verhält es sich mit jedem, der aus dem Geist geboren ist."

Nikodemus ist irritiert und bekommt ergänzend erläutert, dass es zwei Formen des "Geboren-Seins" zu unterscheiden gilt: die aus dem Fleisch und die aus dem Geist, was ihn veranlasst zu fragen, ob und wie man ggf. auch noch als Erwachsener aus dem Geiste geboren werden könne und was das meint.

Die Antwort von Jesus ist, dass man ihm und mit ihm Gott glauben solle, dass den Menschen ein Geboren-Sein aus dem Geiste und aus dem Wasser - also aus der mit dem Leben und den anderen Menschen verbindenden Taufe - dass also den Menschen ein Leben aus dem "Geiste" möglich sei, wenn man an das glaube, was die Taufe will und vermag. Was also gilt es zu glauben? Dass Gott "aus dem Geist" geborene Menschen auf der Erde wolle und Jesus, als der aus dem Geiste geborene MenschenSohn, den Menschen zeige, was es bedeutet, aus dem Geist heraus zu leben und zu handeln.

Eine Gemeinschaft, die tauft, ist vor diesem Hintergrund betrachtet also ein Kreis von Menschen, der an die menschliche Möglichkeit von einem Leben aus dem Geiste heraus glaubt. Eine solche Gemeinschaft empfängt die Neugeborenen als ,Gotteskinder', also als solche, die mit der Möglichkeit ausgestattet sind, aus dem Geiste heraus zu leben. Damit übermitteln die taufenden Gemeinschaften $\mathrm{Zu}-$ versicht an die getauften Neugeborenen, und zugleich übermitteln sie - so lässt sich weiter interpretieren - ein Versprechen: nämlich, dass sie es den durch die Taufe aus dem Geist geborenen Kindern zugestehen, sich so zu verhalten, wie der Wind. Sie erinnern sich: „Der Wind weht, wo er will und du hörst sein Sausen; aber du weißt nicht, woher er kommt, noch wohin er geht". Jesus hatte ja genau das dem Lehrer der Menschen erläutert: „So verhält es sich mit jedem, der aus dem Geist geboren ist". Der Lehrende soll, so lässt sich ,lesen', verstehen: aus dem Geiste lebende Menschen sind unberechenbar, und sie dürfen es sein. Und so heißt Getauft-Werden, in die Gemeinschaft derer aufgenommenen zu sein, die einander zusichern: Es ist in Ordnung, dass ihr euch hinbewegt, wohin ihr es mögt und ihr seid in eurer Nicht-Berechenbarkeit akzeptiert und in unserer Mitte aufgehoben.

Aus erziehungs- und bildungswissenschaftlicher Perspektive ist genau das von zentralem Interesse: Es lässt sich dazu festhalten, dass in der hier umrissenen christlichen Tradition zu denken, bedeuten kann, Menschenkindern eine wirklich günstige Entfaltungsbedingung zu schaffen, insofern darin gilt, 
dass man Kindern/ Menschen ein Leben aus dem Geiste zutraut, und dass man ihnen zusichert, aufgehoben und angenommen zu sein, und zwar ohne Vorbehalt und mit der Bereitschaft verknüpft, sie zu akzeptieren, unabhängig davon, wie sie sich entwickeln. Indem ich so formuliere und interpretiere, stellt sich die Frage, ob diese mögliche Botschaft des Evangeliums nicht im Kern als eine pädagogische Botschaft des Neuen Testaments an die Lehrer der Menschen zu lesen sein könnte.

Die Frage bejahend interessiert mich also jetzt: Wie meint dieser Jesus als Belehrer der Lehrer das genauer? Was ist der Kontext dieses Aufrufs zu einem Einander-ohne-Bedingungen-im-jeweiligenfreien-geistigen-Tun-Annehmen? Was ist das für eine Programmatik? Wie wird sie testamentarisch ausbuchstabiert und was könnte es heißen, sie pädagogisch einlösen zu wollen?

Ich habe mit Blick auf diese Fragen - einmal alternativ zum bildungswissenschaftlichen Diskurs - in der Bibel weiter nachgelesen und betrachte für das fortgesetzte Nachdenken über die Belehrung des Nikodemus durch Jesus den Beginn des Johannes-Evangeliums als eine tragfähige Arbeitsgrundlage: Johannes schafft hier eine - modern gesprochen - erkenntnistheoretische Klärung wenn es heißt:

„Im Anfang war das Wort, und das Wort war Gott, und Gott war das Wort". Gott wird hier, zu Beginn dieses Evangeliums, eingeführt als das Wesen, das "das", was ist, in Worte fasst. "Alles ist durch es das In-Worte-Fassen - geworden, und ohne es ist nichts geworden". Die Folgen sind gravierend: "Was geworden ist" - durch dieses In-Worte-Fassen / durch den Geist / durch Gott - für das gilt: "in ihm war das Leben/ und das Leben war das Licht der Menschen. Und das Licht" - dieses Licht des Wortes auf das, was ist - "scheint in der Finsternis" - als Finsternis, also als dem Nicht-in-Worte-Gefassten „und die Finsternis hat es nicht ergriffen.”

Wird das Gegebene in Worte gefasst, - so die Klärung - wird es zum Leben, und so wird das gefundene Wort zum Licht auf das Gegebene - das Gegebene, das ohne den in Worte fassenden Geist "Finsternis" bleibt.

Das heißt doch wohl - und das ist pädagogisch sehr folgenreich -: nur durch die gedankliche Aneignung der Welt gelingt ein Leben jenseits der Finsternis, also jenseits der unbegriffenen Fülle des Gegebenen und auch jenseits des bloßen Lebens aus dem Fleische, d.h. nur aus den bestehenden Notwendigkeiten.

Diese ,alte' erkenntnistheoretische Position formulieren wir heute neu, z.B. innerhalb des Konstruktivismus. Sie meint dort so wenig wie bei Johannes, dass die realen Gegebenheiten nicht existierten, wenn wir sie nicht gedanklich/ sprachlich repräsentieren. Gemeint ist - so möchte ich sagen - vielmehr: dass und wie wir das Gegebene in Worte fassen und gedanklich repräsentieren, das macht unser Leben aus. Das entscheidet darüber, wie viel Licht in die Finsternis des Erdenlebens kommt: "Das Wort war das wahre Licht, das jeden Menschen erleuchtet, es kam in die Welt" so heißt es bei Johannes. Und dann wird aus "es" im Text ein "er", also Gott. Und er/ Gott macht nach Johannes jetzt eine merkwürdige Erfahrung, nämlich: "Er war in der Welt, und die Welt ist durch ihn geworden, und die Welt hat ihn nicht erkannt".

Mir scheint, das soll aufzeigen: Weil das "In-Worte-Fassen" existiert, existiert die Welt. Aber die Welt bzw. der die Welt schaffende Gott sowie die sie schaffenden Menschen haben nicht in der Hand, dass verstanden wird, dass das so ist. "Er kam in sein Eigentum" - also das in Worte fassende Wesen kommt in die Welt, die seine Worte geschaffen haben, und stellt fest - so erzählt das Johannesevangelium weiter - "die Seinigen nehmen ihn nicht auf". 
Eine schwierige Situation. Und so fragt sich, was macht denn Gott, als das In-Worte-Fassende Wesen? Wir lesen dazu bei Johannes: "Allen aber, die ihn aufnahmen, gab er Macht, Kinder Gottes zu werden". D.h. in meinen Augen: Allen, die verstehen, dass die Welt das Ergebnis des "Gegebenes-in-WorteFassen" ist, gab Gott/ gab das mögliche Wort die ,göttliche' Macht, eben das zu tun, was Gott als Schöpfer, was den Geist, ausmacht: die Welt in Worte zu fassen und damit - wie Gott - Welt zu schaffen.

Das erzählt das Neue Testament und berichtet auch, wie die Menschen von ihren Lehrern eben das lernen können sollten: Gott zeigt ihnen die Möglichkeit dieser Macht, indem er einen Menschen, nämlich Jesus, als denjenigen schickte und benannte, "der nicht aus dem Willen des Fleisches, nicht aus dem Willen des Mannes, sondern aus Gott geboren wurde".

Das Prinzip/ die Option des In-Wort-Fassens und damit des Welt-Schaffen-Könnens geht mit diesem Jesus, der das Prinzip verstanden hat und es den Menschen erklärt, über auf den Menschen als dem Gottessohn, der zugleich der Menschensohn namens Jesu ist: "Und das Wort ist Fleisch geworden und hat unter uns gewohnt." - sagt das Johannes-Evangelium dazu.

Deswegen lässt sich in der christlichen Tradition denken und sagen: Die Menschenkinder sind, wenn sie auf den Namen Jesu getauft werden, aus seinem Geiste, als aus dem Wort/ dem Geist Geborene. So Getaufte sind gottes-ebenbildlich, sie können mit ihren Worten Licht in die Finsternis bringen, wenn sie von ihrer Neu-Geburt aus dem Geiste Gebrauch machen, d.h. wenn sie daran glauben und danach leben.

Ich denke, diese Botschaft Jesu lässt sich durchaus als eine menschheitliche und zugleich pädagogische Herausforderung hören: Sie besteht darin zu verstehen, dass die Welt so wird, wie wir Menschen sie in Worte fassen/ wie wir sie begreifen. Die Vertreter des Konstruktivismus haben eben das formuliert: Die Welt konstruierende Tätigkeit der Menschen gilt es wahrzunehmen, zu verstehen, aufzugreifen und in ihren Konsequenzen zu verantworten (siehe statt anderer: von Förster, 1985).

Als pädagogische Herausforderung daran ist zu verstehen und zu akzeptieren, dass beim Welt-inWorte-Fassen jeder neue Mensch die Möglichkeit hat, diese Welt für sich neu zu begreifen und damit auch zu verändern und dass „Gott“ / das Leben aus dem Geist von den Lehrern der Menschen fordert, dass sie die neuen Menschenkinder im Gebrauch dieser Möglichkeiten, d.h. im Gebrauch ihrer Freiheit zur Selbstbestimmung und zum Welten-Schaffen, annehmen und unterstützen.

Was folgt daraus in erziehungs- und bildungswissenschaftlicher Perspektive?

Wenn die Welt das ist, was wir in Worte gefasst haben, dann kommt es darauf an, dass wir Erwachsenen uns über die dabei getroffenen Entscheidungen Rechenschaft ablegen, um sie den Nachwachsenden offen legen zu können. In dem, wie wir die Welt heute sehen, stehen wir einerseits auf den Schultern von Riesen - wie Norbert Elias sagt -; unser Verstehen ist eine gigantische kollektive Leistung vieler Menschen, die uns und auch kommenden Generationen zu Gute kommen soll. Zugleich und andererseits kann es aber sein, dass die neu ankommenden Menschen womöglich besser als wir, die schon Etablierten, sehen können, dass und wie man intelligenter mit der Welt umgehen könnte, als wir es bisher getan haben und derzeit tun. Das gilt es zuzulassen und zu akzeptieren.

Das gemeinsame Leben im Geiste bedeutet also dreierlei: 
- die Selbstaufklärung der Erwachsenen über die von ihnen in Worte gefasste und damit zu verantwortende Welt,

- die Ermutigung, der Potenzialität der Nachwachsenden zur Formgebung und, der Möglichkeit nach, zur neuen Erfassung und Gestaltung der Welt auf der Erde Raum zu geben sowie schließlich

- die Akzeptanz der Unvorhersehbarkeit und Nicht-Vorbestimmbarkeit der Entwicklung der Welt und der (neuen) Menschen und ggf. der Menschheit.

Alles drei entspricht dem, was in meinen Augen spätestens heute von einer an Selbstbestimmung und Weltentfaltung - also an „Bildung“ - orientierten Pädagogik eingefordert werden sollte.

Es zu leben - in Schulen, Hochschulen, Elternhäusern - ist weder einfach noch erreicht. Es ist nur möglich auf der Basis einer pädagogisch professionellen Haltung, die der Form eines pädagogischen Eides, der immer mal wieder im Gespräch ist (siehe auch v. Hentig, 1997, S. 244-247), einen sinnvollen Gehalt gäbe, womit ein pädagogischer Eid dem Taufversprechen gleichkommen könnte: Eine Zusicherung an die nachwachsende Generation über das von der ,Gemeinde' Getragen-Werden und zu der prinzipiellen Annahme eines jeden als schöpferischem Wesen. Das wäre eine Zusicherung, von der die Menschen für ihre produktive Entwicklung stark unterstützt werden könnten und würden.

Heute belegen auch die Neurowissenschaften das, was Jesus vor 2000 Jahren als Anforderung an die Lehrer der Menschen ,offenbart' hat, und was die wissenschaftliche Pädagogik seit spätestens der Aufklärung eigentlich weiß und doch selbst immer wieder missachtet: Akzeptanz, AngenommenSein, Angstfreiheit und ermutigende, anregende Bedingungen und Angebote zur Auseinandersetzung mit den Herausforderungen des Lebens und der dazu bereits verfügbaren Antworten/ Lösungen machen den Geist tatsächlich frei und beweglich; sie stärken unser aller (menschlich-göttliches) schöpferisches Potenzial, die Welt gedanklich zu erschließen, sie begrifflich zu erfassen und sie verantwortlich, d.h. aus dem Geiste heraus, zu gestalten.

Was folgt aus der so möglichen Rekapitulation des Taufversprechens? Es geht weder im christlichen Kontext noch in diesem Text hier um eine Sonntagsrede. Als Erziehungs- und Bildungswissenschaftlerin, Hochschullehrerin und Erdenbürgerin schlage ich vor, das Gesagte als notwendige Alltagsrede zu lesen und zu denken, also als eine Programmatik des wirklichen Tuns und zwar - wie ich glaube - eines heute dringend erforderlichen Tuns. Warum? Dazu ein paar abschließende Gedanken in den Worten und Theoriebezügen, in denen wir WissenschaftlerInnen und auch ich das Gesagte normalerweise vertreten.

Unser Bemühen, dahinter zu kommen, wie das ,System Erde' - also die ,Schöpfung' - funktioniert, hat uns mit dem ,Essen vom Baum der Erkenntnis' - so der basale jüdisch/ christliche Mythos - aus dem Paradies, als dem Ort des Aufgehobenen-Seins, ,vertrieben'. Wir wurden mit dem ,Essen vom Baum der Erkenntnis' jene schöpferischen Wesen und deshalb wurden wir notwendig aus der paradiesischen Fürsorge entlassen und mussten uns selber kümmern ${ }_{i i}$ Das war/ ist beschwerlich, und wir haben dabei jede Menge Fehler gemacht, die aber vom System und dem darin waltenden Geist über einige Zeit ausgeglichen werden konnten und wurden, weil das System eine hohe Fehlertoleranz hat/ hatte, wie es mit Buckerminster Fuller (wie wir gleich sehen werden) formuliert werden könnte (Fuller, 1976, S. 148/149).

Noch im Rahmen dieser Fehlertoleranz kommt nun dieser Jesus und sagt: Wenn ihr aus dem Geist des Ganzen lebt, seid ihr wie das Ganze, und dann werdet ihr ewig leben, d.h. ihr werdet gemeinsam mit dem Ganzen der Erde Bestand haben. 
Wir haben gehört, dass viele das, was dieser Jesus gesagt hat, vielfach nicht verstanden haben: "Er war in der Welt, und die Welt ist durch ihn geworden, und die Welt hat ihn nicht erkannt."

Wir Menschen hatten seit dieser klärenden, heute quasi wissenschaftlich fundierten ,Offenbarung', ca. 2000 Jahre Zeit u.a. von dem Lehrer Jesus zu lernen. Aber das hat sich so nicht eingestellt und insofern ist irgendwann auch die längste Lernzeit zu Ende und die Menschen müssten erwachsen werden, d.h. sie müssten tatsächlich lernen, selbst Verantwortung zu übernehmen. Der Zeitpunkt dafür ist heute da, wenn nicht schon überschritten: In dem schönen Buch „Bedienungsanleitung für das Raumschiff Erde" hat Buckminster Fuller diesen jetzigen Zustand unserer Menschenwelt bereits 1969 so auf den Punkt gebracht:

"Jetzt jedoch kommen sie aus dem Schoß erlaubter Unwissenheit, in dem sie ihre frühe subjective Phase der Geborgenheit, Versorgung und Beaufsichtigung verbrachten, und sie fangen an, sich all der Dinge, die wir bisher behandelt haben, komprehensiv bewußt zu werden. Sie fangen an zu verstehen, daß sie sich inmitten eines begrenzten, biosphärischen Systems der Lebenserhaltung befinden, dessen ursprünglich überreiche Lebensvorräte nur angelegt waren, um dafür zu sorgen, daß die Menschheit ihre anfänglichen Versuch-und-Irrtum-Entdeckungen ... im Universum machen konnte. Die Menschen kommen schnell zu der Einsicht, daß sie jetzt beginnen müssen, bewusst ihr Raumfahrzeug Erde mit totaler planetarischer Kooperation, Kompetenz und Integrität zu lenken (Fuller, [1969], S. 148/149)."

Nun könnte man aus der vorstehenden Rekonstruktion und Interpretation der Tauftradition folgern: Die Menschen müssten und würden zu dieser Einsicht kommen, wenn sie aus dem Geiste lebten. Eigentlich müssten die Gemeinschaften, die Menschen taufen, Gemeinschaften von Menschen sein, die verstanden haben, dass die Welt erhalten/ gerettet werden kann und die Menschen „ewiges Leben“ haben, wenn sie dem gesandten Gottessohn als dem Lehrer der Lehrenden glauben würden und danach handelten, was er sagt.

Der zu vermittelnde Glauben besteht darin, für möglich zu halten, dass sich die Welt durch ihre gedankliche Durchdringung als lebensfähig und überlebensfähig für alle Menschen einrichten ließe. Bedingung für die Wirksamkeit dieses Glaubens ist der entsprechende Umgang mit den bestehenden geistigen Erschließungen der Welt und mit den neuen Menschen, so dass diese einerseits möglichst umfassend und klar in den Stand der menschheitlich erzielten Klärungen eingeführt werden und andererseits Gelegenheit und Ermutigung erhalten, mit diesen Klärungen neu umzugehen. In pädagogischer Sprache: Es geht um die Ermöglichung von "Bildung" als der Befähigung zum selbstbewussten und selbstbestimmten Umgang mit gesellschaftlichen Wissensbeständen bezogen auf die menschheitlich zu bearbeitenden Aufgaben (siehe dazu ausführlicher: Girmes, 2012), und es geht um das Fördern der Bereitschaft, Verantwortung zu übernehmen für das, was die gewählten gedanklichen Fassungen der Welt mit der realen Welt machen.

Die Erziehungs- und Bildungswissenschaftler und Bildungswissenschaftlerinnen sehe ich also dazu herausgefordert, den Menschen/ Kindern beim selbstbewussten und selbstbestimmten Umgang mit sich und der Welt mit gesellschaftlichen Wissensbeständen zur Seite zu stehen. Es ist vielleicht eine ungewohnte Sichtweise auf die Aufgabe/n dieser Wissenschaft/en, aber ich betrachte sie als dazu aufgerufen, ihre Arbeit in eben diesem Geiste zu verstehen und auszuüben: Es ist diese Art pädagogischer Eid denkbar, als ein Bemühen um die Einlösung des Taufversprechens, als eines Versprechens der Erwachsenengeneration an die Nachwachsenden. Das Versprechen bestände darin, die Wirksamkeit des Geistes wahrzunehmen und auch anzunehmen, d.h., der Freiheit der Menschen und dem Gebrauch dieser Freiheit Anlass und Raum zu geben und zu lassen. Das ergibt und ereignet sich 
nämlich nicht von selbst, weil es meint, der menschlichen Möglichkeit zur freien Entfaltung beim ,Der-Erde-und-dem-Leben-auf-ihr-Form-Geben' die dazu erforderlichen Fähigkeiten und Haltungen zugänglich und ergreifbar werden zu lassen.

Es kann helfen und dazu beizutragen, diesen großen Auftrag zu realisieren, wenn man sich vorzustellen versucht, dass eine Schule, eine Hochschule, ja jede Bildungseinrichtung wie eine ,Gemeinde' gestaltbar wäre, in der so gelebt werden kann und gelebt wird, dass dort spürbar ist: Die ,Gemeindemitglieder' wissen um die Macht und weltschaffende Wirksamkeit der gedanklichen Durchdringung und Formung der Welt. Und sie respektieren die entsprechenden Potenziale all ihrer Mitglieder, die sie als Formgebende schätzen und fördern - und haben dabei mit besonderer Aufmerksamkeit die Potenziale ihrer Kinder und aller sich entfaltend lernenden Mitglieder im Blick.

Ich finde in diesem Zusammenhang, dass viel stärker ins Bewusstsein zu heben wäre, dass es dafür, in dieser Weise mit der nachwachsenden Generation und allen bildungsaktiven Menschen umzugehen, einen attraktiven „Lohn“ gibt, nämlich die Partizipation an geistigem Wachstum überhaupt. Für mich steht deshalb der andere Lohn für eine entsprechende Haltung, den das Johannesevangelium wir kommen darauf nochmals kurz zurück - anbietet, eher nicht im Vordergrund. Er sei trotzdem erwähnt:

"Wer an ihn glaubt, wird nicht gerichtet; wer nicht glaubt, ist schon gerichtet, weil er an den Namen des eingeborenen Sohnes Gottes nicht geglaubt hat. Darin aber besteht das Gericht, dass das Licht in die Welt gekommen ist und die Menschen die Finsternis mehr liebten als das Licht; denn ihre Werke waren böse. Denn jeder, der Schlechtes tut, hasst das Licht und kommt nicht zum Licht, damit seine Werke nicht aufgedeckt werden. Wer aber die Wahrheit tut, der kommt zum Licht, damit seine Werke offenbar werden, dass sie in Gott getan sind."

Sicher geht es auch darum, am Ende als Mensch und als für die menschliche Entfaltung auf jeden Fall Mitverantwortlicher "gerechtfertigt" dazustehen. (M)Eine Art der Verantwortungsfrage gerecht zu werden, ist allerdings eher die der Teilhabe an der Freude über die lebensbezogene und lebensbejahende Botschaft, die Jesus, der hier vorgestellte Lehrer der Menschen, übermittelt: Wir sind aufgefordert und explizit gerechtfertigt, uns unseres Geistes für das Leben zu bedienen. Es geht darum, sich selbst und andere im Gebrauch des/ ihres Geistes zu fördern: Das ist eine, vielleicht sogar die große menschliche Herausforderung und Aufgabe, nicht zuletzt, sondern zu allererst für die Bildungswissenschaften. Ob die angemessene Bearbeitung dieser Aufgabe in der knapper werdenden Zeit noch gelingen wird und kann, ist mittlerweile fraglich. Es zu versuchen, die Arbeit angemessen in Angriff zu nehmen, erscheint mir aus der Liebe zur Welt, zu den Menschen und zum Leben angezeigt.

Alternativ zum Johannesevangelium aber gleichsinnig lässt sich das mit Hannah Arendt auch so formulieren:

„In der Erziehung entscheidet sich, ob wir die Welt genug lieben, um die Verantwortung für sie zu übernehmen und sie gleichzeitig vor dem Ruin zu retten, der ohne Erneuerung, ohne die Ankunft von Neuen und Jungen, unaufhaltsam wäre. Und in der Erziehung entscheidet sich auch, ob wir unsere Kinder genug lieben, um sie weder aus unserer Welt auszustoßen und sich selbst zu überlassen, noch ihnen ihre Chance, etwas Neues, von uns nicht Erwartetes zu unternehmen, aus der Hand zu schlagen, sondern sie für ihre Aufgabe der Erneuerung einer gemeinsamen Welt vorzubereiten" (Arendt, [1968] 1994, S. 273).

Es ist sicher gut, wenn Menschen sich diese gemeinsam vorzubereitende und zu schaffende Welt als eine vorstellen können, die aufgehoben ist in der Welt Gottes/ des Wortes/ des Geistes. Wenn die 
Menschen das können und tun, dann besteht - so darf man sagen - berechtigte Hoffnung auf den Bestand der Welt, und es ist nicht mehr so schwierig, die Unbestimmtheit auszuhalten - wovor wir Erwachsenen nicht selten die meiste Angst haben. Es ist dann akzeptierbar, nicht zu wissen, wie eine Welt mit der neuen Generation und mit mehr Licht in der Finsternis - also mit mehr aufklärendem Wissen - aussehen wird.

Die Taufe und die Taufgemeinschaft als Prototyp für die Einladung zu ,Bildung' und in Bildungsgemeinschaften könnten zeigen, wie aus dieser Angst vor der Unbestimmtheit und Ungewissheit eine Zuversicht werden kann - etwas, das der Pädagogik und ihren Wissenschaften bisher nur mit mäßigem Erfolg zu bewirken gelungen ist. Die trotz Unsicherheit mögliche und berechtigte Zuversicht basierte auf der Gewährleistung eines Raumes mit der Zusicherung, dass darin Aufgehobenheit ohne Vereinnahmung bestehen und gelebt werden. Die durch einen solchen Raum begründbare Zuversicht wäre - wie sich mit Nikodemus vom ,Lehrer' Jesus und in der Beschäftigung mit dem Johannesevangelium auf neue Weise lernen ließe -, ein mögliches und ein heute fast notwendiges Geschenk der gottebenbildlichen Menschen an sich selbst, an den Geist und an die Welt, die ja durch formgebende Wesen geschaffen wurde. Man kann/ muss hoffen, dass es viele Gemeinschaften geben wird, die dieses Geschenk der Aufgehobenheit ohne Vereinnahmung, also in noch anderen Worten und auch anderen möglichen Traditionen ausgedrückt, das Geschenk der Liebe, zu machen verstehen. Mir scheint auch jenseits der christlichen Tradition argumentierbar, dass wir viele solcher Bildungsgemeinschaften entstehen lassen sollten und dass wir sie brauchen werden, wenn der Menschheit ein dauerhaftes Leben auf der Erde möglich bleiben soll. Die Zukunft der Bildung und ihrer Wissenschaften, das ist meine These (Girmes, 2012, Kap I.4), würde damit erst zu einer Übernahme der ihr sich stellenden und gestellten Aufgaben in einer Welt kommen, in der die Menschen hoch dringlich die Verantwortung für die Formgebung ihres Geistes übernehmen müssten. Mir scheint: Es gilt, Bildung und Bildungseinrichtungen in eben diesem Sinne neu zu erfinden. Das kann man - wie zu zeigen war - im Anschluss an die obige Deutung des Sinns der christlichen Taufe denken und tun; aber die damit verbundene Argumentationsfigur trägt aus meiner Sicht auch unabhängig davon. So lade ich ein, ihr zu folgen.

\section{Literatur}

Arendt, Hannah ([1968] 1994): Zwischen Vergangenheit und Zukunft. Übungen im politischen Denken. München. Zürich: Piper Verlag.

Fromm, Erich ([1976] 2006/2007): Haben oder Sein. Die seelischen Grundlagen einer neuen Gesellschaft. Aus dem Engl. von Brigitte Stein. Stuttgart: Deutsche Verlags-Anstalt. (genutzt: Ungekürzte Lizenzausgabe des SPIEGEL-Verlags. Hamburg).

Fuller, Richard Buckminster ([1969] 1998): Bedienungsanleitung für das Raumschiff Erde und andere Schriften. Amsterdam, Dresden: Verlag der Kunst.

Girmes, Renate (2012): Der Wert der Bildung. Menschliche Entfaltung jenseits von Knappheit und Konkurrenz. Paderborn: Verlag Schöningh.

Foerster, Heinz von (1985): „Konstruieren einer Wirklichkeit.“ In: Paul Watzlawick (Hg.): Die erfundene Wirklichkeit: Wie wissen wir, was wir zu wissen glauben? Beiträge zum Konstruktivismus. München. Zürich: Piper Verlag.

Hentig, Hartmut von (1993): Die Schule neu denken. München/Wien: Beltz-Verlag.

(Die hier in Klammern stehenden Jahreszahlen sollen da, wo es mir bedeutsam erscheint, in Erinnerung bringen/ halten, seit wann ein Text/Konzept/Gedanke publiziert vorliegt.) 


\section{Über die Autorin}

Prof. em. Dr. Renate Girmes: Lehrgebiete: Bildungstheorie, Aufgabenorientierte Didaktik, Theorie von Bildungsräumen und Bildungssettings; Professorin für Allgemeine Didaktik und Theorie der Schule; Bildungsunternehmerin und Geschäftsführerin von Odyssee's End GmbH \& Co. KG. Kontakt: renate.girmes@odyssees-end.de

i Grundlage des Textes ist eine Predigt von Renate Girmes, gehalten in einem Akademischen Gottesdienst in Magdeburg (URL:www.ekmd.de/attachment/aa234c91bdabf36adbf227d333e5305b/b797debe4de611dd83b54f7212dec81ec81e/Predigt+R._Girmes.pdf)

ii Dass und wie das u.a. im Anschluss an Erich Fromm (1976) genauer zu denken sein könnte, habe ich an anderer Stelle ausführlich dargestellt, in: Girmes, 2012, S. 52-74. 